\section{Improved Surface Treatment of the Superconducting TESLA Cavities ${ }^{1}$}

\author{
L. Lilje ${ }^{2}$, A. Matheisen, D. Proch, \\ D. Reschke, D. Trines, \\ DESY, Notkestrasse 85, D-22607 Hamburg, Germany \\ C. Antoine, J.-P. Charrier, H. Safa, B. Visentin, \\ CEA Saclay, DAPHNIA, 91191 Gif-sur-Yvette, France \\ C. Benvenuti, D. Bloess, E. Chiaveri, L. Ferreira, R. \\ Losito, H. Preis, H. Wenninger \\ CERN, CH-1211 Geneva 23, Switzerland \\ P. Schmüser \\ Universität Hamburg, Notkestrasse 85, D-22607 \\ Hamburg, Germany
}

\begin{abstract}
The proposed linear electron-positron collider TESLA is based on $1.3 \mathrm{GHz}$ superconducting niobium cavities for particle acceleration. For a center-of-mass energy of 500 $\mathrm{GeV}$ an accelerating field of $23.4 \mathrm{MV} / \mathrm{m}$ is required which is reliably achieved with a niobium surface preparation by chemical etching. An upgrade of the collider to 800 $\mathrm{GeV}$ requires an improved cavity preparation technique. In this paper results are presented on single-cell cavities which demonstrate that fields of up to $40 \mathrm{MV} / \mathrm{m}$ are accessible by electrolytic polishing of the inner surface of the cavity.
\end{abstract}

Keywords: Superconducting RF cavities, Niobium, Surface treatments, Accelerating gradients, High-energy accelerators

PACS: 74.25.Nf, 74.60.Ec, 81.65.Ps, 84.70.+P, 29.17.+W

\section{Introduction}

Linear electron-positron colliders for physics in the Higgs and supersymmetric particle regime have to reach centre-of-mass energies in excess of $500 \mathrm{GeV}$, and hence high accelerating fields in the accelerating structures are needed to limit the size of the accelerator complex. TESLA [1] is the only collider project based on superconducting cavities. In the $500 \mathrm{GeV}$ baseline design (TESLA-500) the accelerating field, often called "gradient" hereafter, amounts to $23.4 \mathrm{MV} / \mathrm{m}$. The $1.3 \mathrm{GHz}$ nine-cell niobium cavities for the TESLA Test Facility (TTF) linac have achieved an average gradient of $26.1 \pm$ $2.3 \mathrm{MV} / \mathrm{m}$ at a quality factor $Q_{0} \geq 1 \cdot 10^{10}$ in the most recent industrial production of 24 cavities. The technology

\footnotetext{
${ }^{1}$ Article published in Nucl. Inst. Meth. A 516 (2-3) (2004) pp.213-227., DESY 2004-017, TESLA 2004-04

${ }^{2}$ Corresponding author: Lutz.Lilje@desy.de
}

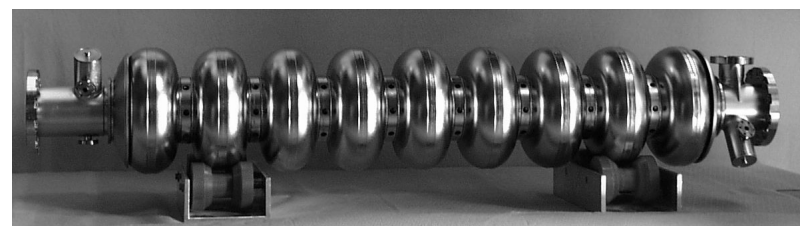

Figure 1: Superconducting $1.3 \mathrm{GHz}$ 9-cell cavity for the TESLA Test Facility.

developed for TTF is hence adequate for TESLA-500 but considerable improvements are needed for an upgrade of the collider to $800 \mathrm{GeV}$ (TESLA-800). A detailed description of the present status of the nine-cell cavity layout, fabrication, preparation and tests can be found in $[2]$.

The TESLA cavities (Figure 1) are made from $2.8 \mathrm{~mm}$ thick niobium sheets by deep drawing and electron beam welding. A damage layer of about $100 \mu \mathrm{m}$ thickness has to be removed from the inner surface to obtain an optimum performance in the superconducting state. For the TTF cavities this has been done so far by chemical etching, see below. Using an alternative preparation method, electrolytic polishing (or electropolishing for short), scientists at the KEK laboratory in Tsukuba (Japan) have achieved gradients of up to $40 \mathrm{MV} / \mathrm{m}$ in $1.3 \mathrm{GHz}$ singlecell cavities [3]. This was the motivation for starting an R\&D program on electropolishing of single-cell cavities of the TESLA shape. This program has been carried out in collaboration between CERN, DESY and Saclay.

\section{Niobium Chemistry}

Niobium metal has a natural $\mathrm{Nb}_{2} \mathrm{O}_{5}$-layer with a thickness of about $5 \mathrm{~nm}$. Below this layer other oxides and sub-oxides can be found $[4,5,6] . \mathrm{Nb}_{2} \mathrm{O}_{5}$ is chemically rather inert and can be dissolved only with hydrofluoric acid (HF). The strong acids used in the niobium surface treatment like $\mathrm{HF}, \mathrm{HNO}_{3}$ or $\mathrm{H}_{2} \mathrm{SO}_{4}$ play also an essential role for removing defects from the inner surface of the cavity either by dissolving the foreign material itself or the surrounding niobium. Such defects may be abrasive particles from grinding, imprints from the deep drawing process, niobium protrusions from scratches or dirt particles sticking to the surface. Cleaning the niobium with chemical methods is the most practical way to achieve a high quality superconducting surface. Chemical or electrolytic processes can be applied to niobium resonators if the safety and environmental standards regarding HF-containing acid mixtures are obeyed. Nitrous gases, oxygen and hydrogen are produced during etching or electropolishing. A review of the preparation methods of niobium cavities is given in [7]. 


\subsection{Chemical etching}

The sheet rolling of niobium produces a damage layer of about $100 \mu \mathrm{m}$ thickness which has to be removed in order to obtain a surface with excellent superconducting properties. The standard procedure is chemical etching which consists of two basic steps: dissolution of the $\mathrm{Nb}_{2} \mathrm{O}_{5}$ layer by $\mathrm{HF}$ and re-oxidation of the niobium by $\mathrm{a}$ strongly oxidizing acid $[8,9]$. Then the new oxide layer will be dissolved by the HF again. The most frequently used oxidizing agent for niobium is nitric acid $\left(\mathrm{HNO}_{3}\right)$. The material removal rate in a mixture of $\mathrm{HF}(40 \%)$ and $\mathrm{HNO}_{3}(65 \%)$ is large, of the order of $30 \mu \mathrm{m}$ per minute [9]. The reactions are strongly exothermic and can cause a thermal runaway situation. Additionally, large quantities of gases (hydrogen, nitrous gases, HF) are produced. For these reasons the mixture of $\mathrm{HF}$ and $\mathrm{HNO}_{3}$ alone is not suitable for the etching of cavities.

To obtain a better process control a buffer substance like phosphoric acid $\mathrm{H}_{3} \mathrm{PO}_{4}$ (concentration of $85 \%$ ) may be added [10]. Furthermore the mixture is cooled below $15^{\circ} \mathrm{C}$ which also reduces the migration of hydrogen into the niobium lattice $[9,11]$. The standard procedure with a removal rate of about $1 \mu \mathrm{m}$ per minute is called buffered chemical polishing ${ }^{3}$ (BCP) with an acid mixture containing 1 part $\mathrm{HF}, 1$ part $\mathrm{HNO}_{3}$ and 2 parts $\mathrm{H}_{3} \mathrm{PO}_{4}$ in volume. Another possibility is to use concentrated sulphuric acid $\left(\mathrm{H}_{2} \mathrm{SO}_{4}\right)$ as a buffer $[9,12]$. At TTF, a closed-circuit chemistry system is used in which the acid is pumped from a storage tank through a cooling system and a filter into the cavity and then back to the storage. The gases produced are not released into the environment without prior neutralization and cleaning. The cavities are rinsed with low pressure ultrapure water immediately after the chemical treatment.

By now there exists compelling evidence that the BCP process limits the attainable accelerating fields of multicell cavities to about $30 \mathrm{MV} / \mathrm{m}$ even if niobium of excellent thermal conductivity is used. Typical accelerating gradients achieved with BCP in defect-free cavities are shown in figure 2. Measurements on etched onecell cavities in other laboratories yielded similar results $[13,14,3]$. Etched cavities exceeded $30 \mathrm{MV} / \mathrm{m}$ only in rare cases $[15,16]$.

The etching process is accompanied with undesirable effects such as migration of hydrogen into the bulk niobium and strong grain boundary etching. The first effect can be reduced by cooling the acid below $15^{\circ} \mathrm{C}$ [11]. In principle, grain boundary etching could be suppressed by using an acid mixture with a high etching rate. This, however, would not be advisable in the nine-cell TESLA cavity with an inner surface of about $1 \mathrm{~m}^{2}$ since the large amount of produced heat would speed up the reaction even more and preclude a well-controlled etching process.

\footnotetext{
${ }^{3}$ The term "polishing" is not really adequate since the resulting surface roughness is in the $\mu \mathrm{m}$ range and grain boundaries are enhanced.
}

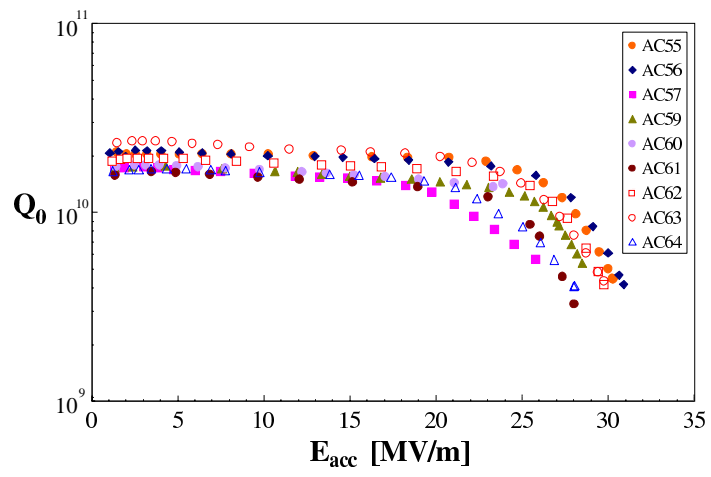

Figure 2: Excitation curves of several nine-cell TESLA cavities with surface preparation by chemical etching (BCP). The quality factor is plotted as a function of the accelerating field. Test temperature is $2 \mathrm{~K}$.

\section{$2.2 \quad$ Electrolytic polishing}

An alternative method to etching is electrolytic polishing or electropolishing (EP) in which the material is removed in an acid mixture under the flow of an electric current. Sharp edges and burrs are smoothed out and a very glossy surface can be obtained. The electric field is high at protrusions so these will be dissolved first. On the other hand, the field is low in the grain boundaries and little material will be removed here while in the BCP process strong etching is observed in the boundaries between grains.

Electropolishing of niobium cavities has been known for 30 years. The most widely used electrolyte is a mixture of concentrated $\mathrm{HF}$ and concentrated $\mathrm{H}_{2} \mathrm{SO}_{4}$ in volume ratio of 1:9 [17]. A pulsed electric current was used in a horizontal EP setup for superconducting niobium cavities prepared at CERN in collaboration with Karlsruhe in 1979 [18]. A continuous method for horizontal EP has been developed at KEK in 1989 [19].

The chemical processes are as follows $[7,20]$ :

$$
\begin{array}{rll}
2 \mathrm{Nb}+5 \mathrm{SO}_{4}^{--}+5 \mathrm{H}_{2} \mathrm{O} \rightarrow & \mathrm{Nb}_{2} \mathrm{O}_{5}+10 \mathrm{H}^{+} \\
& +5 \mathrm{SO}_{4}^{--}+10 \mathrm{e}^{-} \\
\mathrm{Nb}_{2} \mathrm{O}_{5}+6 \mathrm{HF} \rightarrow & \mathrm{H}_{2} \mathrm{NbOF}_{5} \\
& +\mathrm{NbO}_{2} \mathrm{~F} \cdot 0.5 \mathrm{H}_{2} \mathrm{O}+1.5 \mathrm{H}_{2} \mathrm{O} \\
\mathrm{NbO}_{2} \mathrm{~F} \cdot 0.5 \mathrm{H}_{2} \mathrm{O}+4 \mathrm{HF} \rightarrow & \mathrm{H}_{2} \mathrm{NbF}_{5}+1.5 \mathrm{H}_{2} \mathrm{O}
\end{array}
$$

Most systems for the EP of accelerator cavities are horizontal as shown in figure 3 . The advantage is that the produced gases (mainly hydrogen) are rapidly removed from the wetted niobium surface [18]. Gas bubbles sticking on the surface could lead to etching pits. In a vertical setup these bubbles would move slowly upwards and create axial wells. A drawback of the horizontal arrangement is that the cavity has to be rotated. There is some difficulty to achieve a leak tight rotary sleeve for the acid mixture. In addition, the removal rate is reduced by a factor of two since the surface is immersed only half of the time in the acid to allow the hydrogen gas to escape through the upper part of the beam tube. 


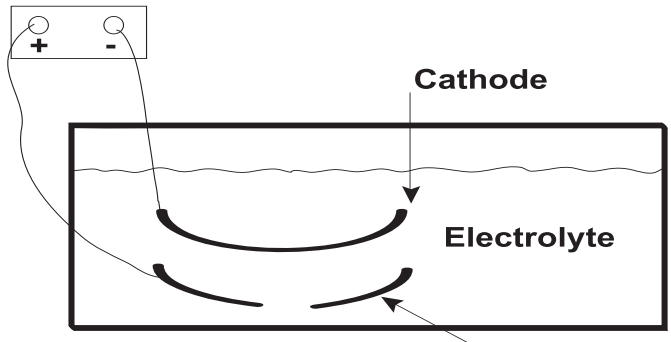

Niobium half cell

a)

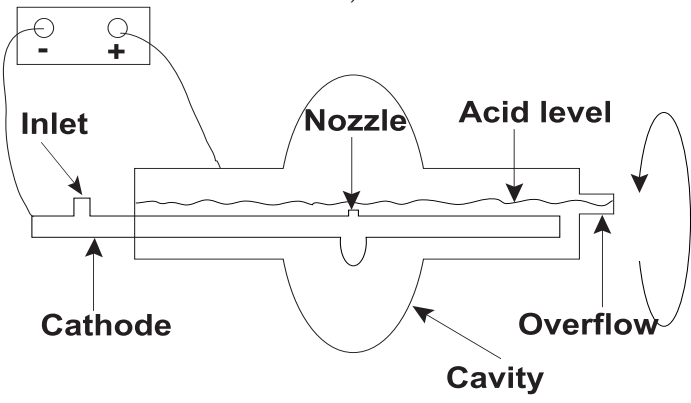

b)

Figure 3: a) Schematic of a half-cell EP system. b: Schematic of a cavity EP system. Detailed descriptions of the two systems are given in the text.

\subsection{Comparison of etched and elec- tropolished surfaces}

Micro-graphs of BCP and EP treated niobium samples are compared in figure 4 . One can see that EP smoothes out the grain boundaries far better than BCP. The average roughness of chemically etched niobium surfaces is of the order of $1 \mu \mathrm{m}$ [12] while EP surfaces are at least one order of magnitude smoother, see figure 5. It should be noted, however, that the measured unevenness of the surface depends on the scan length of the atomic force microscope. On a nanometer scale the roughness of BCP and EP treated samples is comparable. This can be understood because at such small lengths the measurement takes place on a single niobium grain. The main difference between EP and BCP is the smoothening of the ridges at grain boundaries which is measured only when the scan length is sufficiently large.

On etched surfaces the step height at grain boundaries can be a few $\mu \mathrm{m}$. Particularly large steps may occur at the electron-beam welds at the cavity equator. For nonoptimized weld parameters steps as high as $30 \mu \mathrm{m}$ have been observed [21]. The steps at grain boundaries and in the weld seams may lead to a magnetic field enhancement and a premature breakdown of superconductivity [22]. This interpretation is corroborated by the observation that several chemically etched cavities exhibited a quench in the region of high magnetic field near the equator weld without any indication of contamination by foreign materials. It was possible to shift the quench position along the equator region by applying a new chem-

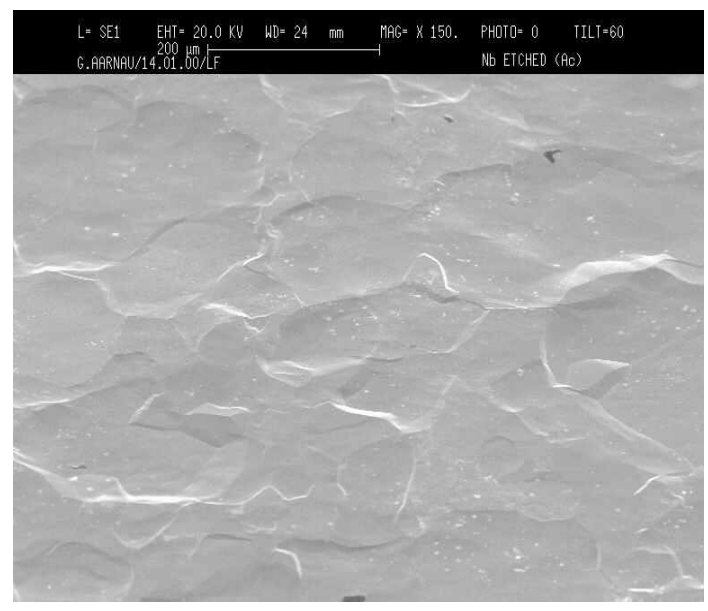

a) $400 \times 800 \mu \mathrm{m}$

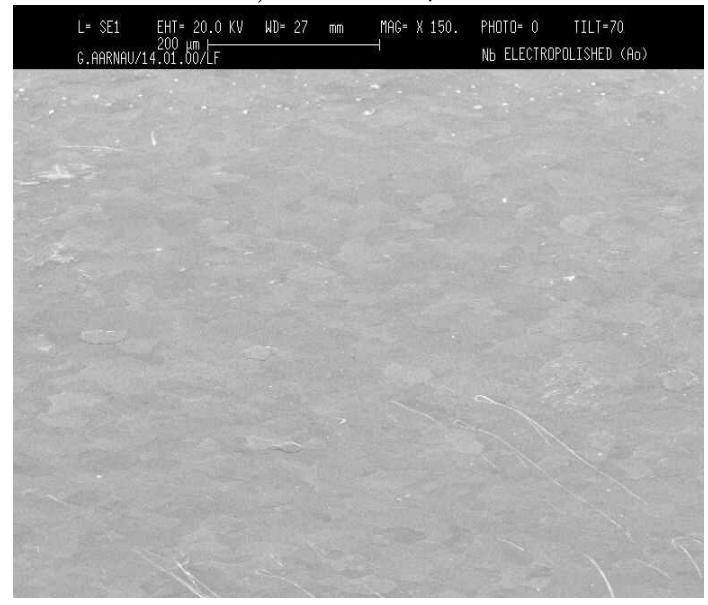

b) $400 \times 800 \mu \mathrm{m}$

Figure 4: Niobium surfaces after etching (a) and electropolishing (b). SEM micro-graphs are courtesy of G. Arnau, CERN.

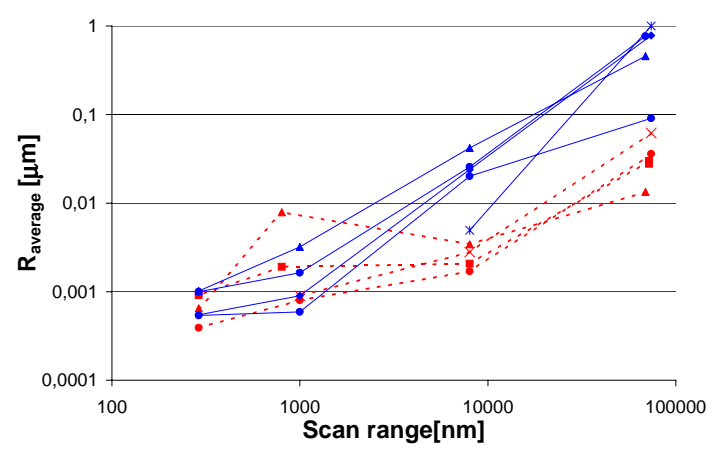

Figure 5: Average roughness as a function of the scan length of an atomic force microscope. EP treated samples are shown with dotted lines, while BCP samples are shown with full lines. At large scan length EP yields about one order of magnitude lower average roughness than $B C P$ while for a short scan length (less than the grain size) the difference becomes smaller. 
ical etching, but the maximum achievable field was not improved [23, 24].

An important question for electropolishing is of course how much material has to be removed to achieve a smooth surface. On electropolished samples the roughness drops below $1 \mu \mathrm{m}$ after $150 \mu \mathrm{m}$ of material have been removed. This corresponds to the thickness of the damage layer which has to be removed anyway. Therefore an electropolishing of at least $100 \mu \mathrm{m}$ is a reasonable choice both for surface smoothening and damage layer removal.

\subsection{Description of the EP systems}

\subsubsection{Half-cell electropolishing}

In the framework of the DESY-CERN-Saclay collaboration a setup for the EP of half-cells was developed at CERN. The goal was to investigate the viability of a novel cavity fabrication and preparation concept: removal of most of the damage layer by EP on simple subunits and assembly of the multi-cell cavity from prepolished half-cells. An electrolyte developed at CERN $[25,26]$ was used (see table 1) with a removal rate of $1-2 \mu \mathrm{m}$ per minute. The electrode was made from copper. An electropolishing of about $100 \mu \mathrm{m}$ was applied to the half cells. Then 4 one-cell cavities were electronbeam welded at an industrial company with a standard electron-beam welding apparatus (vacuum $10^{-5}$ mbar), and 11 cavities were welded at CERN under much better vacuum conditions (pressure in the welding chamber below $10^{-6}$ mbar).

Two of the cavities were tested immediately after welding and high-pressure water rinsing. Not unexpectedly, the results were poor: the cavities reached a maximum accelerating field of just $8-10 \mathrm{MV} / \mathrm{m}$ with a strong decrease in quality factor. A slight chemical etching which would have been sufficient to dissolve a possible copper deposition on the niobium surface cured the Q-degradation, however electron field emission was still present at low excitation implying that another contaminant besides copper was present. After a chemical etching (BCP) of $20-50 \mu \mathrm{m}$ both cavities reached the standard performance $(25 \mathrm{MV} / \mathrm{m})$ of BCP-treated cavities. The obvious explanation is that welding deposits on the inner surface of the cavity must be removed by a BCP or EP of $20-50 \mu \mathrm{m}$ to arrive at a defect-free surface.

The conclusion to be drawn from these observations is that the fabrication of a multi-cell cavity from prepolished half-cells is indeed a viable concept which would substantially reduce the amount of EP needed in the finished resonator.

\subsection{Electropolishing of complete cavities}

A setup for the electropolishing of single-cell cavities was built at CERN. The parameters were chosen similar to those of the successful KEK system [19] and are summarized in table 1 . The essential difference is the cathode

\begin{tabular}{|c|c|}
\hline $\begin{array}{l}\text { EP for half cells } \\
\text { Acid mixture }\end{array}$ & $\begin{array}{ll}24 \% & \mathrm{HF}(40 \%) \\
21 \% & \mathrm{H}_{2} \mathrm{SO}_{4}(96 \%) \\
38 \% & \mathrm{H}_{3} \mathrm{PO}_{4}(83 \%) \\
17 \% & \text { Butanol }\end{array}$ \\
\hline $\begin{array}{l}\text { Voltage } \\
\text { Current density } \\
\text { Removal rate }\end{array}$ & $\begin{array}{l}4-6 \quad \mathrm{~V} \\
1-1.4 \quad \mathrm{~A} / \mathrm{cm}^{2} \\
1-2 \quad \mu \mathrm{m} / \mathrm{min}\end{array}$ \\
\hline $\begin{array}{l}\text { EP for cavities } \\
\text { Acid mixture }\end{array}$ & $\begin{array}{ll}10 \% & \mathrm{HF}(40 \%) \\
90 \% & \mathrm{H}_{2} \mathrm{SO}_{4}(96 \%)\end{array}$ \\
\hline $\begin{array}{l}\text { Voltage } \\
\text { Current density } \\
\text { Removal rate } \\
\text { Temperature of electrolyte } \\
\text { Rotation } \\
\text { Acid flow }\end{array}$ & $\begin{array}{l}15-20 \mathrm{~V} \\
0.5-0.6 \quad \mathrm{~A} / \mathrm{cm}^{2} \\
0.5 \mu \mathrm{m} / \mathrm{min} \\
30-35 \quad{ }^{\circ} \mathrm{C} \\
1 \quad \mathrm{rpm} \\
5 \quad \text { liters } / \mathrm{s}\end{array}$ \\
\hline
\end{tabular}

Table 1: Parameters for the EP of half-cells and the EP of one-cell cavities at CERN.

material: pure aluminum at KEK and copper at CERN. Both materials have their respective advantages and disadvantages. The copper electrode requires some care, see below. The cathode is surrounded with tube made from a porous $\mathrm{PTFE}^{4}$ cloth to keep the electrolytically produced hydrogen gas away from the niobium surface.

Except for the cathode all components of the EP system are made from plastic material which is inert against the aggressive acid mixture. All parts in contact with the $\mathrm{HF}$ should be made from fluoroplastics such as $\mathrm{PFA}^{5}$, $\mathrm{PVDF}^{6}$ or PTFE. The EP system is placed in a vented area where the exhaust gases are pumped through a neutralization system to avoid environmental hazards. The acid mixture is contained in a closed-circuit. The electrolyte is stored in containers cladded with Teflon which can be cooled by water flowing through Tefloncovered piping. The volume above the electrolyte is filled with dry nitrogen to avoid water vapour absorption by the strongly hygroscopic $\mathrm{H}_{2} \mathrm{SO}_{4}$. The acid mixture is pumped with a membrane pump through a cooler and a filter with $1 \mu \mathrm{m}$ pore size before it reaches the inlet of the cathode. Then the electrolyte flows through the cathode to the center of the cell (see figure 3(b)). The acid returns to the storage tank via an overflow.

The cavity is installed horizontally together with the cathode and then the lower half is filled with the electrolyte which attacks the niobium only very slowly when no voltage is applied (etch rate less than $1 \mathrm{~nm}$ per hour). After the equilibrium filling level has been reached, the rotation is switched on and a leak check is done. Then the current is switched on and the current-voltage characteristic is measured. At a certain voltage (see table 1) current oscillations set in which are an indication that two alternating processes take place: dissolution of $\mathrm{Nb}_{2} \mathrm{O}_{5}$ by $\mathrm{HF}$ and re-oxidation by $\mathrm{H}_{2} \mathrm{SO}_{4}$. The best pol-

\footnotetext{
${ }^{4}$ Polytetrafluoroethylene, for example Teflon $\mathbb{R}$

${ }^{5}$ Polyperfluoroalkoxyethylene

${ }^{6}$ Polyvinylidene Fluoride
} 
ishing results are obtained for an oscillation amplitude of $10-15 \%$ around the mean value. The temperature of the acid has to be around $30-35^{\circ} \mathrm{C}$ during the EP. Temperatures above $40^{\circ} \mathrm{C}$ must be avoided as they result in etching pits on the surface.

When the desired amount of material has been removed, the current is switched off. The rotation is stopped and the cavity is put into vertical position to release the acid mixture. After rinsing with pure water the cavity and the electrode are dismounted. Another low-pressure water rinsing follows and the wet cavity is taken into a glove-box with nitrogen atmosphere. Here the cavity is rinsed with an ultrapure high-pressure water jet to remove remaining chemical residues. A rinsing with filtered ethanol follows to speed up the drying process. The cavity is then stored overnight in a vacuum of $10^{-3}$ mbar. Then the cavity is either rinsed with highpressure water for the performance tests at CERN or filled with nitrogen gas and sent to Saclay or DESY for high-pressure water rinsing and tests.

It should be mentioned that the copper electrode used in the CERN system has a disadvantage. While the current is flowing, the copper becomes passivated but after the current has been switched off copper ions can be dissolved and precipitate on the cavity surface. It has been observed in a test that this leads to an increased residual resistance and to strong field emission at low field $(<10 \mathrm{MV} / \mathrm{m})$ [27]. To remove the copper deposition the cavities are rinsed in sequence with $\mathrm{HNO}_{3}(60 \%)$, water, $\mathrm{HF}$ and water again. These steps are repeated twice. The $\mathrm{HNO}_{3}$ dissolves the copper and oxidises the niobium. Hydrofluoric acid removes the oxide layer and several contaminants as indicated by our surface studies. The overall material removal by this procedure is only about $10 \mathrm{~nm}$ and does not deteriorate the smoothness of the electropolished surface.

\section{Measurements on electropol- ished cavities}

\section{1 $\quad$ First tests}

In their first tests the cavities which were electropolished in the CERN system showed an unexpected performance limitation: the excitation curves exhibited a strong degradation in quality factor at high field as can be seen in figure 6 . Field emission of electrons could be excluded as an explanation for the performance degradation since neither $\mathrm{X}$ rays nor secondary electrons were observed. Temperature mapping revealed a global heating in the areas of high magnetic fields around the equator indicating a rapid increase of the microwave surface resistance towards high microwave fields.

In contrast to this observation, the excitation curves of the EP-treated cavities at KEK showed only a moderate drop of the quality factor and reached much higher accelerating fields, see figure 7 [28, 3, 24].

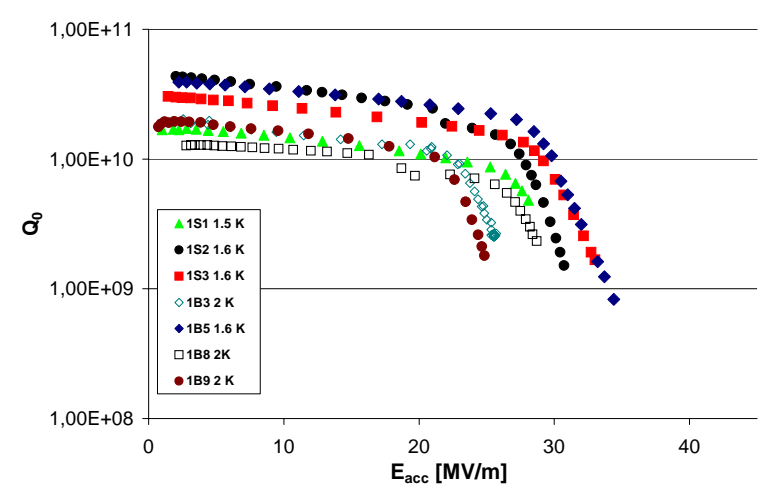

a)

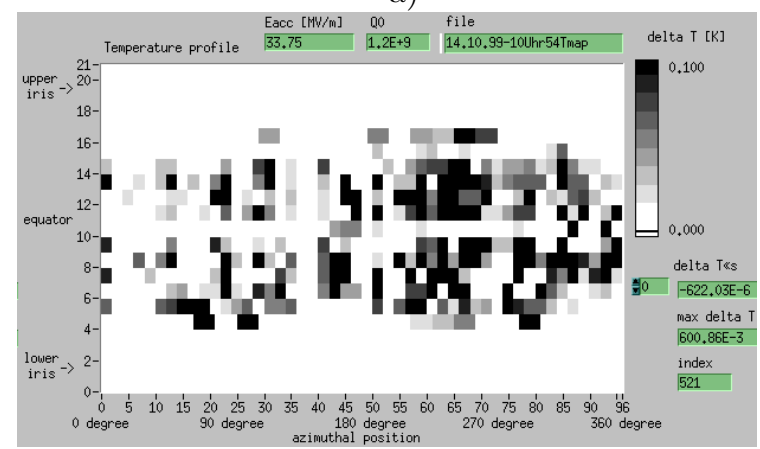

b)

Figure 6: a) First tests of electropolished single-cell resonators. Note the strong degradation of the quality factor at accelerating fields $E_{a c c}=25-30 \mathrm{MV} / \mathrm{m}$. b) Temperature map of an electropolished single-cell resonator. Shown is an unwrapped view of the outer cavity surface. The heating of the surface (dark spots) takes place near the equator in the region high magnetic field.

At Saclay a similar strong $Q$ degradation was observed for chemically etched cavities $[23,14,29]$, see figure 8 . The temperature mapping revealed a global heating of the surface similar to that in figure $6 \mathrm{~b}$. It was discovered that the unsatisfactory performance could be considerably improved by applying a moderate thermal treatment to the finished cavity [29]. This will be called bakeout (or in situ bakeout) hereafter. The procedure at Saclay was as follows. After the last high-pressure water rinsing the cavities were evacuated and then heated up to $170^{\circ} \mathrm{C}$ for 70 hours. The remarkable observation was that this low-temperature baking improved the quality factor at the highest field by nearly a factor of 3 . This result was confirmed in other tests on BCP-treated cavities. However, tests revealed also that the baking procedure did not increase the maximum field level in chemically etched cavities [29]. 


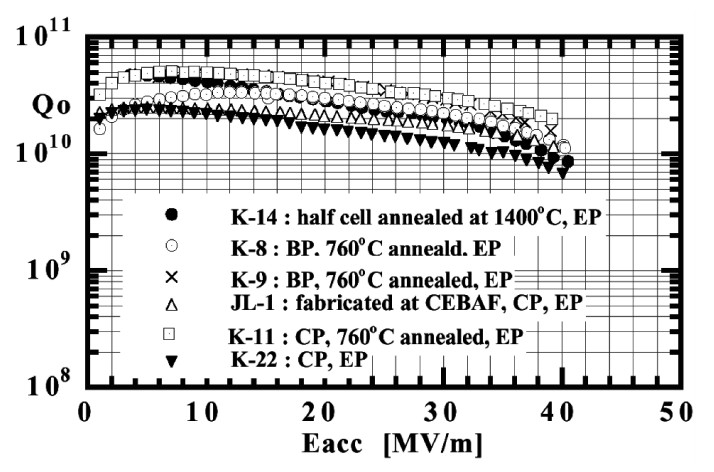

Figure 7: Results from electropolished single cell resonators at KEK [24]. No evidence of a strong degradation of the quality factor is seen. As part of the standard preparation at KEK a low-temperature baking at $85-120^{\circ} \mathrm{C}$ was applied to the evacuated cavities. Test temperature was $1.5-1.7 \mathrm{~K}$. (Courtesy K. Saito, E. Kako)

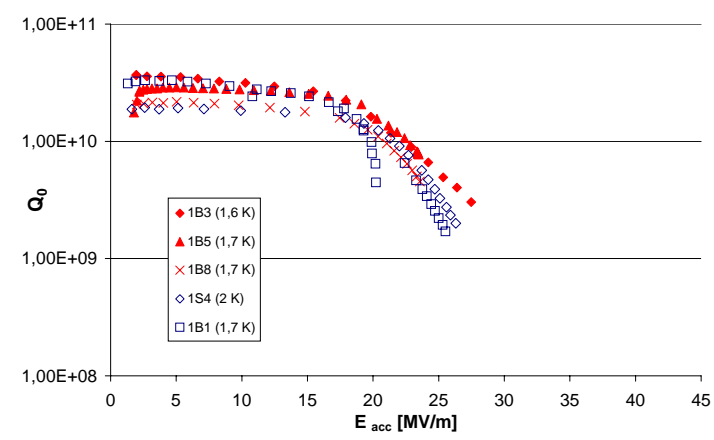

a)

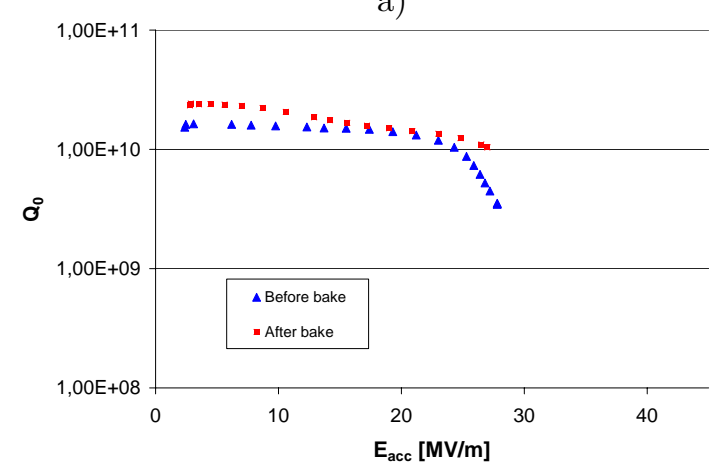

b)

Figure 8: a) Excitation curves of etched single cell resonators showing a strong degradation of the quality factor at $E_{a c c}=25 \mathrm{MV} / \mathrm{m}$. b) Influence of the bakeout procedure on the quality factor $Q_{0}$ of a $B C P$-treated cavity. Test temperature was $2 \mathrm{~K}$.

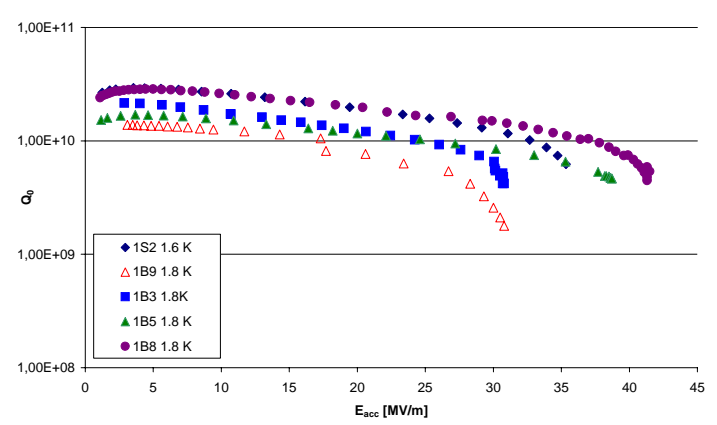

Figure 9: Excitation curves of electropolished one-cell cavities after the low-temperature bakeout. The tests have been performed under slightly different conditions (different magnetic shielding in test cryostats and temperature).

\subsection{Application of low-temperature bakeout to EP cavities}

Building on the experience with BCP cavities at Saclay and with EP cavities at KEK (where the bakeout at 85$100^{\circ} \mathrm{C}$ had been part of the standard preparation) it was decided to apply the bakeout to the EP cavities of the CERN-DESY-Saclay collaboration. Figure 9 shows that a dramatic improvement in performance is achieved: several single cell cavities reach now accelerating gradients of up to $40 \mathrm{MV} / \mathrm{m}$ with quality factors above $5 \cdot 10^{9}$. This behaviour is very similar to the observations made at KEK.

The heat treatment of niobium cavities is a well-known method to improve the performance. The temperature is in general quite high though: the TTF cavities are heat treated in an UHV furnace at $800^{\circ} \mathrm{C}$ for hydrogen degassing and stress annealing and afterwards at $1400^{\circ} \mathrm{C}$ for increasing the thermal conductivity of the bulk niobium $^{7}$. The surprising observation made with the bakeout effect is that a thermal treatment at such a low temperature, where the diffusion of any gases dissolved in the niobium lattice is extremely slow, has a major influence on the high-gradient performance. It it obvious that only the thin surface layer which is essential for the microwave superconductivity can be modified by the bakeout.

\subsection{High-field performance at different helium temperatures}

Figure 10 shows the excitation curves of an electropolished cavity before and after the bakeout at helium bath temperatures between $1.5 \mathrm{~K}$ and $2.2 \mathrm{~K}$. At low field the quality factor exhibits the well-known temperature dependence which is caused by the exponential temperature dependence of the BCS surface resistance (see below). However, the maximum achieved gradient and the corresponding $Q_{0}$ value are almost independent of the

\footnotetext{
${ }^{7}$ During this furnace treatment a titanium getter layer is evaporated onto the niobium surface which protects the niobium from being oxidized by the residual oxygen in the furnace atmosphere.
} 
bath temperature as long as the helium coolant is in the superfluid state. These results are consistent with thermal model calculations [30]. Only when the temperature is in the vicinity of the Lambda-point $(2.17 \mathrm{~K})$ of liquid helium, where the transition from the superfluid to the normal-fluid phase takes place, a degradation of the quality factor due to insufficient cooling can already be seen at low field.

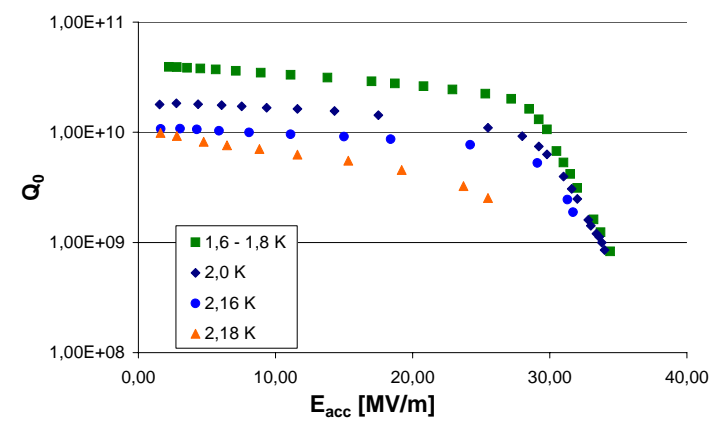

a)

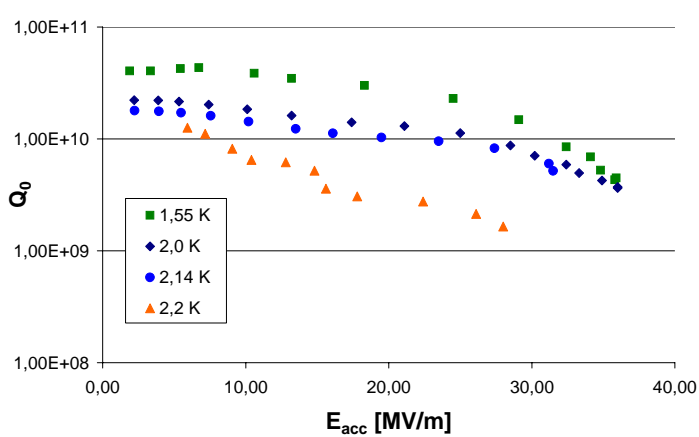

b)

Figure 10: Excitation curves of an electropolished cavity before (a)) and after bakeout (b)) for different liquid helium temperatures.

\subsection{High temperature heat treatments of electropolished cavities}

Electrolytic polishing generates hydrogen which can penetrate into the niobium lattice. If too much hydrogen is dissolved the danger exists that niobium hydride compounds are formed if the material is exposed to temperatures around $100 \mathrm{~K}$ for an extended period. These $\mathrm{Nb}-\mathrm{H}$ compounds have very high microwave losses and may lead to a reduction of the quality factor by one or two orders of magnitude. This unfortunate effect has been named $Q$-disease.

The typical hydrogen concentration in etched niobium is in the order 100 - 400 atomic ppm hydrogen in the bulk material and 4 atomic percent in a $50 \mathrm{~nm}$ surface layer $[11,31]$. A high temperature treatment at $800^{\circ} \mathrm{C}$ is very effective in reducing the hydrogen concentration to 3 atomic ppm in the bulk and $\leq 1$ atomic $\%$ in the surface layer.
The hydride formation depends on the dwell time the cavity spends in the dangerous region around $100 \mathrm{~K}$. With a fast cooldown from $300 \mathrm{~K}$ to $4.2 \mathrm{~K}$ within 1 hour, hydride formation will be reduced to an acceptable level.

The cavities studied in this work have been made from electropolished half-cells. In the half-cell EP system the niobium is not protected from the hydrogen gas by a membrane. Therefore the danger of quality factor degradation was to be expected. A dedicated experiment was carried out to this end, the results are shown in figure 11. The electropolished and baked cavity was tested after a fast cooldown and showed the usual good performance. Then the cavity was exposed to a temperature of $100 \mathrm{~K}$ for 2 days. In the new test the quality factor was very low and showed the typical strong field dependence described in [11]. In the next step the cavity was heated in a vacuum furnace to $800^{\circ} \mathrm{C}$ to remove the hydrogen from the bulk material. A short electropolishing of about $20 \mu \mathrm{m}$ was done to clean the surface from dirt which might have been introduced during the furnace treatment. Again the cavity was tested after fast cooldown and showed the initial good performance. The exposure of the cavity to the dangerous temperature of $100 \mathrm{~K}$ for 2 days was repeated. This time, however, no $Q$ degradation was observed, indicating that the formation of hydrides was strongly suppressed by the furnace treatment. This is an important and encouraging result which implies that no hydrogen will be introduced into the niobium during a short EP if precautions are taken (PTFE-cloth around the cathode) to keep the gas away from the niobium surface.

Seven cavities received the furnace treatment at $800^{\circ} \mathrm{C}$ after electropolishing. Figure 12 shows the maximum gradients before and after the $800^{\circ} \mathrm{C}$ treatment. The average gradient rises from $35 \mathrm{MV} / \mathrm{m}$ to $35.4 \mathrm{MV} / \mathrm{m}$.

Finally, one electropolished cavity was subjected to the $1400^{\circ} \mathrm{C}$ furnace treatment with titanium getter which is part of the standard preparation of the TTF cavities (see $[2])$. The idea was to check whether the increased thermal conductivity would have a similar beneficial effect on EP cavities as on BCP cavities. The residual resistivity ratio RRR increased from 300 to 500 . The cavity reached the same maximum field of $35 \mathrm{MV} / \mathrm{m}$ as before the $1400^{\circ} \mathrm{C}$ treatment, but at a slightly higher $Q_{0}$. More tests are needed to decide whether the $1400^{\circ} \mathrm{C}$ furnace treatment would constitute a significant improvement of the electropolished multi-cell cavities of the TESLA-800 collider.

\subsection{Comparison of etching and elec- tropolishing}

The test results on etched single-cell cavities are consistent with the performance achieved in the TESLA nine-cell cavities. The average gradient achieved in the one-cell cavities without any heat treatment is $24 \mathrm{MV} / \mathrm{m}$. If one evaluates the single-cell performance of those 


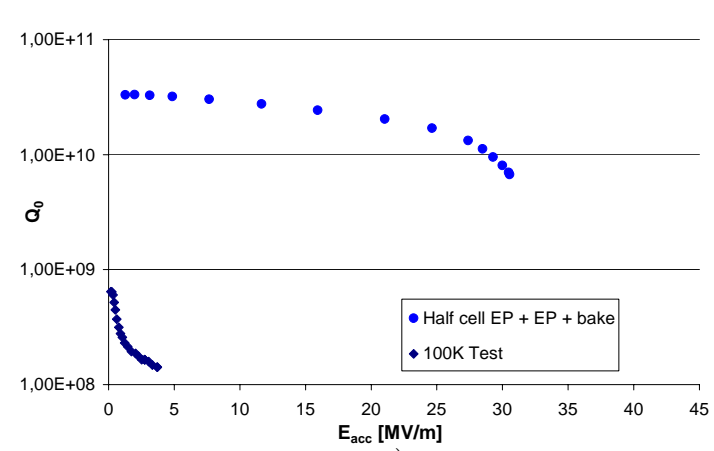

a)

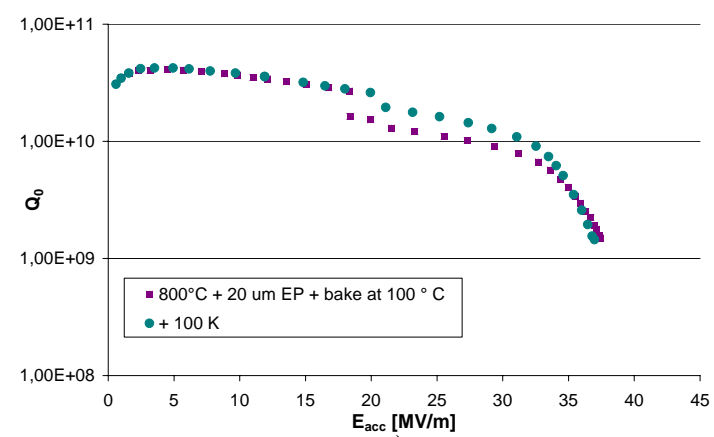

b)

Figure 11: Demonstration of the "Q disease" i.e. the strong degradation due to the formation of niobium hydrides. a) The excitation curve of an electropolished and baked cavity before and after a two-day exposure to a temperature of $100 \mathrm{~K} . \mathrm{b})$ The same cavity after an $800^{\circ} \mathrm{C}$ furnace treatment and a short EP of $20 \mu \mathrm{m}$. The quality factor degradation does not reappear when the cavity is exposed to $100 \mathrm{~K}$. Test temperature $1.6 \mathrm{~K}$.

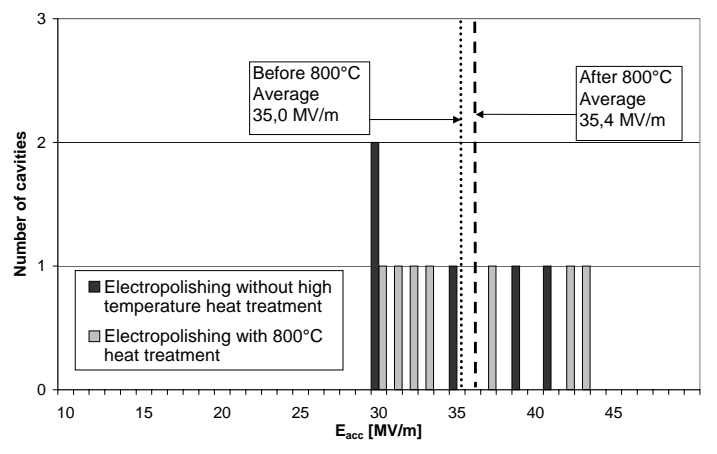

Figure 12: Distribution of accelerating gradients before and after the furnace treatment at $800^{\circ} \mathrm{C}$. One cavity with a weld defect has been omitted from this graph.

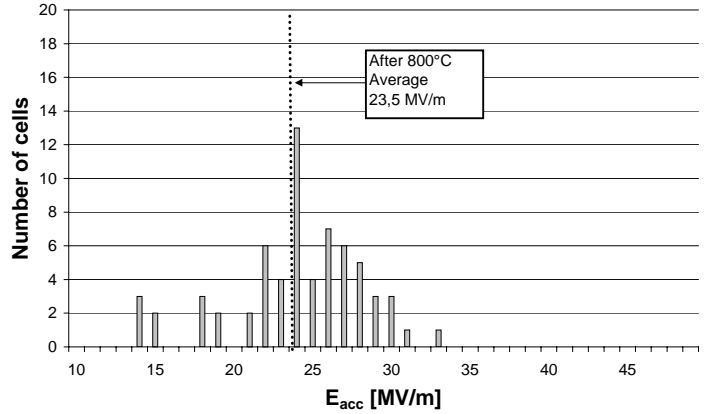

a)

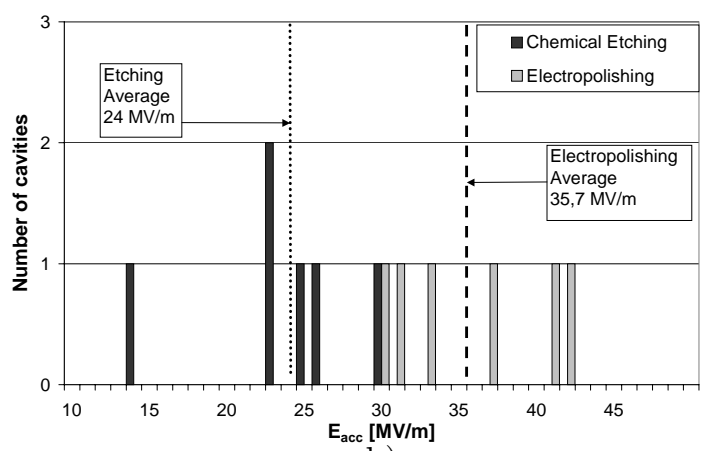

b)

Figure 13: a) Single-cell performance of the nine-cell TESLA cavities after the $800^{\circ} \mathrm{C}$ heat treatment. The average gradient is $23.5 \mathrm{MV} / \mathrm{m}$. b) Distribution of the maximum accelerating gradients of etched and electropolished single-cell cavities. For etched cavities the average gradient is $24 \mathrm{MV} / \mathrm{m}$, for electropolished cavities $35.7 \mathrm{MV} / \mathrm{m}$.

etched nine-cell cavities which were tested after the $800^{\circ} \mathrm{C}$ heat treatment an average gradient of $23.5 \mathrm{MV} / \mathrm{m}$ is obtained. The $1400^{\circ} \mathrm{C}$ heat treatment which is routinely applied to the BCP-treated TTF cavities raises the average gradient to more than $25 \mathrm{MV} / \mathrm{m}$ [2].

A summary of the performance after etching and electropolishing is given in figure 13. The average gradient of the electropolished cavities is $35.7 \mathrm{MV} / \mathrm{m}$. This is convincing evidence that electropolishing leads to substantially higher accelerating fields. One reason is certainly that magnetic field enhancements at grain boundaries are avoided. Moreover, the smoother EP surface contains fewer defects and is easier to clean from chemical residues.

\subsection{Influence of the electron-beam weld- ing on the cavity performance}

The TESLA cavities are made from deep-drawn niobium half-cells which are joined by electron-beam (EB) welding. The quality of the EB weld seam at the equator is crucial for the maximum field achievable in the cavities. The one-cell production was made in two different EB welding machines. The first batch of 4 cavities was produced by an industrial company with experience in 


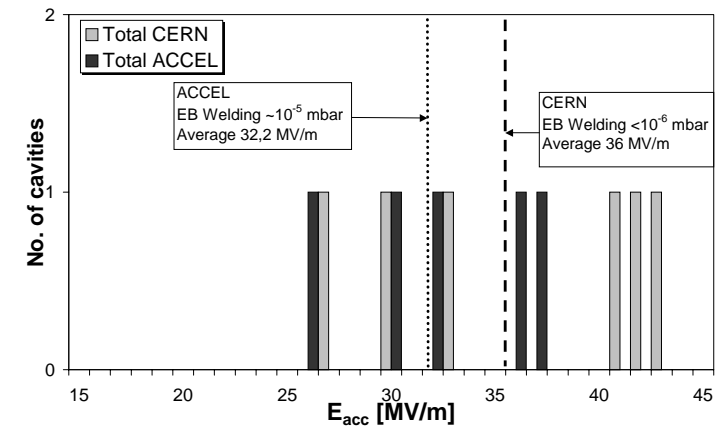

Figure 14: Comparison of accelerating gradients of cavities welded under standard vacuum conditions $\left(\approx 10^{-5} \mathrm{mbar}\right)$ by the company ACCEL and under improved vacuum conditions $\left(<10^{-6}\right.$ mbar $)$ in the electron beam welding machine at CERN. The data for etched and electropolished cavities are shown. The best test on each cavity is taken. One cavity of the CERN batch had a macroscopic defect on a weld seam, which was found during visual inspection. This cavity has been omitted from the figure.

niobium cavity welding (ACCEL Instruments, BergischGladbach). The second batch of 11 cavities was welded at CERN with an EB welding machine equipped with a stainless steel vacuum chamber conforming to ultra high vacuum standards. Whereas the vacuum in the industrial production is of the order of a few times $10^{-5}$ mbar, the pressure in the CERN system could be lowered down to $3 \cdot 10^{-7}$ mbar by using a cryopump and a longer time for pumping down.

As the residual gas in the machine is partly gettered by the molten niobium in the weld seam, the residual resistivity ratio RRR in this region can be degraded. For an initial $\mathrm{RRR} \approx 300$ the reduction can be of the order of $20 \%$ for a vacuum of $10^{-5} \mathrm{mbar}^{8}$. It can be expected that the better vacuum conditions lead to an improved cavity performance. The average gradient of the CERN batch is in fact $3.8 \mathrm{MV} / \mathrm{m}$ higher as compared to the cavities prepared under standard vacuum conditions (see figure 14) ${ }^{9}$.

The CERN EB machine is equipped with a $60 \mathrm{kV}$ electron gun without the possibility to raster the electron beam in a rhombic pattern across the weld which produces the smoothest underbead of the weld seam $[1,33]$. The results in figure 14 indicate that the electron-beam welding facility should combine a state-of-the-art electron gun and a UHV vacuum system. Such an electronbeam welding facility is presently being commissioned at DESY.

\footnotetext{
${ }^{8}$ For TIG (Tungsten-Inert-Gas) welds the reduction can be very large $(\mathrm{RRR} \approx 400$ is reduced to $\mathrm{RRR} \approx 120$ ) [32]. In addition, defects can be introduced using this welding technique.

${ }^{9} \mathrm{All}$ cavities were treated in a vacuum furnace at $800^{\circ} \mathrm{C}$ to remove hydrogen from the niobium.
}

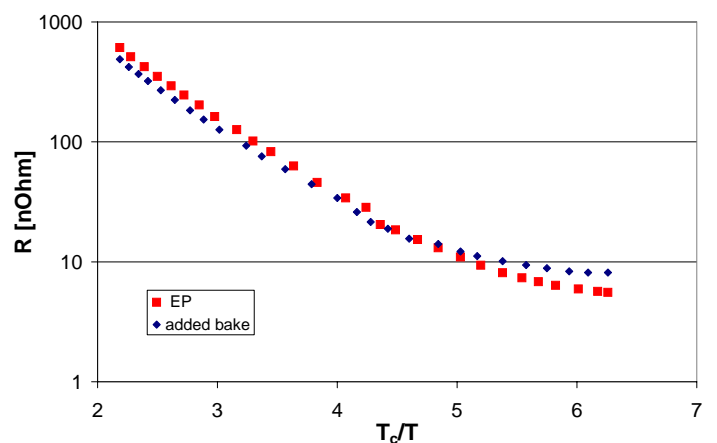

a)

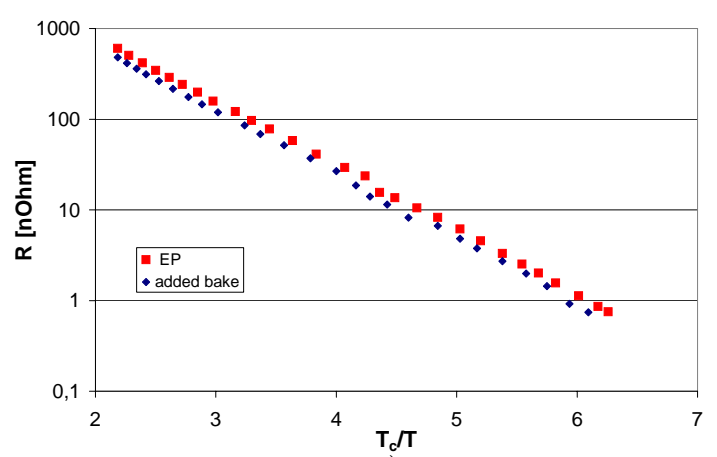

b)

Figure 15: a) Temperature dependence of the surface resistance before and after bakeout of an EP cavity. b) The BCS surface resistance. The residual resistances of $4.7 \mathrm{n} \Omega$ for the unbaked cavity and of $7.4 \mathrm{n} \Omega$ for the baked cavity have been subtracted.

\subsection{Influence of bakeout on the mi- crowave surface resistance}

In figure 15 the microwave surface resistance before and after the low-temperature bakeout is plotted against the inverse temperature. According to the BCS (Bardeen-Cooper-Schrieffer) theory the surface resistance should drop exponentially when plotted against $T_{c} / T$. For temperatures $T<\frac{T_{c}}{2}$ and an energy of the microwave photons $h f$ much less than the energy gap $\Delta$, the surface resistance can be approximated by:

$$
R_{B C S}(T, f)=A \frac{f^{2}}{T} \exp \left(-\frac{\Delta}{k_{B} T}\right)
$$

The factor $A$ depends on material parameters like the coherence length $\xi$, the electron mean free path $\ell$, the Fermi velocity $v_{F}$ and the London penetration depth $\lambda_{L}$. The BCS resistance of niobium at $1.3 \mathrm{GHz}$ is about $600 \mathrm{n} \Omega$ at $4.2 \mathrm{~K}$ and $10 \mathrm{n} \Omega$ at $2 \mathrm{~K}$.

A refined expression, derived from the two-fluid model of superconductors, is [34]:

$$
R_{B C S}(T, f) \propto \sigma_{n c} \cdot \lambda_{e f f}^{3} \frac{f^{2}}{T} \exp \left(-\frac{\Delta}{k_{B} T}\right)
$$

where $\sigma_{n c}$ is the conductivity due to the normalconducting component and $\lambda_{\text {eff }}=\lambda_{L} \sqrt{1+\xi / \ell}$. The 
BCS resistance is hence proportional to the mean free path $\ell$ of the normal-conducting electrons and does not assume its minimum for extremely pure niobium $(\ell$ very large) but rather for somewhat "dirty" $\mathrm{Nb}$ with $\ell \approx \xi$. If we make the assumption that the bakeout creates a "dirty" surface layer with a shorter mean free path than in the bulk niobium, then the residual resistance will be larger and the BCS resistance will be lower than in the unbaked cavity. Figure 15 shows that this is indeed the case. The BCS part of the surface resistance is reduced by a factor of $\approx 1.5$ due to the bakeout while the residual resistance increases. Similar observations were made by Kneisel [35].

It should be noted that the resistance data are derived from measurements of the cavity quality factor at low field (a few $\mathrm{MV} / \mathrm{m}$ ). The peculiar high-field behaviour of unbaked cavities, namely the strong $Q$ degradation, cannot be explained in terms of the BCS resistance because this quantity is independent of the magnitude of the electromagnetic field in the cavity. On the contrary, a strongly field-dependent resistance would be needed to account for the rapid decrease of $Q_{0}$ towards large gradients. This effect is not yet understood.

\section{Conclusions and outlook}

Electropolished bulk niobium cavities offer accelerating fields of more than $35 \mathrm{MV} / \mathrm{m}$ and are hence suitable for the upgrade of the TESLA collider to $800 \mathrm{GeV}$. The surface preparation by electropolishing has a definite advantage in comparison with chemical etching: a much smoother surface is obtained and the field enhancement at grain boundaries is avoided. This magnetic field enhancement may be the origin of a local premature breakdown of superconductivity in etched cavities. The physical and physico-chemical principles of the bakeout effect are not well understood yet and require further research. Meanwhile, the first multi-cell cavities have been electropolished in a collaboration between KEK and DESY. The results will be published in a separate paper.

\section{Acknowledgments}

The authors would like to express their gratitude to O. Aberle, S. Ehmele, S. Forel, A. Lava, A. Insomby, R. Guerin, M. Kubly, the DESY group MKS3, K. Twarowski, J.-P. Poupeau, Y. Gasser for their invaluable help in measurements, cavity fabrication, chemical treatments and handling of cavities.

We are particularly grateful to K. Saito and E. Kako for their advice and information on electropolishing. We would like to thank E. Haebel for the discussions on RF superconductivity and help with the measurement system at CERN. We would like to thank P. Kneisel for stimulating discussions on the bakeout effect.

\section{References}

[1] R. Brinkmann, K. Flöttmann, J. Rossbach, P. Schmüser, N. Walker, and H. Weise, editors. TESLA - Technical Design Report, volume II. DESY, March 2001. DESY 2001-011, ECFA 2001209, TESLA Report 2001-23.

[2] B. Aune et al. The Superconducting TESLA Cavities. Phys. Rev. ST-AB, 3(9), September 2000. 092001.

[3] K. Saito et al. Superiority of Electropolishing over Chemical Polishing on High Gradients. In Palmieri [37], pages $759-813$.

[4] M. Grundner. Oberflächenuntersuchungen an Niob für supraleitende Resonatoren mittels der Röntgen-Photoelektronenspektroskopie und der Auger-Elektronenspektroskopie. $\mathrm{PhD}$ thesis, Universität Karlsruhe, 1977.

[5] M. Grundner and J. Halbritter. XPS and AES studies on oxide growth and oxide coatings on niobium. J. Appl. Phys., 51(1):397-405, 1980.

[6] A. Dacca, G. Gemme, L. Mattera, and R. Parodi. XPS analysis of the surface composition of niobium for superconducting RF cavities. Appl. Surf. Sci., 126:219-230, 1998 .

[7] P. Kneisel. Surface preparations of niobium. In M. Kuntze, editor, Proceedings of the Workshop on RF Superconductivity, volume I+II, page 27, Karlsruhe, 1980. KFK. KFK-3019.

[8] Gmelin. Handbuch der anorganischen Chemie, volume 49(Nb). Springer Verlag Berlin, 1970.

[9] B. Hillenbrand, N. Krause, K. Schmitzke, and Y. Uzel. Abschlussbericht - Supraleitende Resonatoren. Technical Report NT 2024 7, Siemens AG, Dezember 1982. BMBF Forschungsbericht.

[10] J. Guerin. Etude du bain de polissage chimique de niobium. Technical Report TE/LC/157/89, CERN, October 1989.

[11] R. Röth. Untersuchungen zu anomalen Verlusten in Niobresonatoren. PhD thesis, Universität Wuppertal, 1993. WUP-DIS 92-12.

[12] C. Z. Antoine et al. Alternative approaches for surface treatment of $\mathrm{Nb}$ superconducting cavities. In Krawcyk [36], pages 109-117.

[13] P. Kneisel. A collection of higher gradient cavity experiments. In Palmieri [37], pages 830-842.

[14] H. Safa. High gradients in scrf cavities. In Palmieri [37], pages $814-821$. 
[15] P. Kneisel, R. Röth, and H.-G. Kürschner. Results from a nearly "defect-free" niobium cavity. In P. Bonin, editor, Proceedings of the 7th Workshop on RF Superconductivity, volume I+II, pages 447453, Gif-sur-Yvette, October 1995. IN2P3.

[16] B. Visentin, J-P. Charrier, A. Aspart, Y. Gasser, J-P. Poupeau, and G. Congretel. A Nonelectropolished Niobium Cavity Reached $40 \mathrm{MV} / \mathrm{m}$ at Saclay. In Proceedings of EPAC 2002, pages 2292-2294, 2002.

[17] H. Diepers, O. Schmidt, H. Martens, and F.S. Sun. A new method of electropolishing niobium. Physics Letters, 37A(2):139, 1971.

[18] A. Citron, G. Dammertz, M. Grundner, L. Husson, R. Lehm, and H. Lengeler. The Karlsruhe-CERN superconducting RF separator. Nucl. Inst. Meth., 164:31 - 55, 1979 .

[19] K. Saito et al. Electropolishing of L-band cavities. In Y. Kojima, editor, Proceedings of the 4 th Workshop on RF Superconductivity, volume I+II, pages 635 - 695, Tsukuba, 1989. KEK. KEK Report 8921.

[20] L. Ponto and M. Hein. Elektropolitur von Niob. Technical Report WUP 86-17, Bergische Universität Wuppertal, 1986.

[21] R.L. Geng, J. Knobloch, and H. Padamsee. Microstructures of RF surfaces in the electron beam-weld regions of niobium. In Krawcyk [36], pages 238-245.

[22] J. Knobloch, M. Liepe, R.L. Geng, and H. Padamsee. High-Field Q slope in Superconducting Cavities Due to Magnetic Field Enhancement at Grain Boundaries. In Krawcyk [36], pages 77-91.

[23] E. Kako et al. Cavity performance in the 1,3 GHz Saclay/KEK cavities. In Palmieri [37], pages 491502.

[24] E. Kako et al. Improvement of Cavity Performance in the Saclay/Cornell/Desy's sc Cavities. In Krawcyk [36], pages 179-186.

[25] J.P. Birabeau and J. Guerin. Electrolytic polishing of $\mathrm{Nb}$ and Ti. Technical Report W 33, CERN, March 1974.

[26] J.P. Birabeau and J. Guerin. Additif a l'étude de bain du polissage électrolytique du niobium formulé en Juin 1974. Technical Report SB/AC/B/3035/gp, CERN, March 1982.

[27] L. Lilje. Experimental Investigations on Superconducting Niobium Cavities at Highest Radiofrequency Fields. PhD thesis, Universität Hamburg, 2001. DESY-THESIS-2001-034.
[28] K. Saito et al. High accelerating gradients in niobium L-band cavities. Particle Accelerators, 60:193$217,1997$.

[29] B. Visentin, J.P. Charrier, and B. Coadou. Improvements of superconducting cavity performances at high gradients. In Proceedings of the 6th EPAC, volume III, page 1885, 1998.

[30] D. Reschke. Thermal model calculations for 1,3 GHz TTF accelerator cavities. In Palmieri [37], page 385.

[31] B. Bonin and R. Röth. Q degradation of niobium cavities due to hydrogen contamination. In D. Proch, editor, Proceedings of the 5th Workshop on RF Superconductivity, volume I+II, pages 210 244, Hamburg, April 1992. DESY. DESY-M-92-01.

[32] D. Proch, W. Singer, and H. M. Wen. Some Results about RRR Distribution in Nb of Superconducting Cavities for the TESLA Test Facility. TESLA Report TESLA 98-02, DESY, 1998.

[33] H. Padamsee, J. Knobloch, and T. Hays. RF Superconductivity for Accelerators. John Wiley \& Sons, 1998.

[34] B. Bonin. Materials for superconducting cavities. In S. Turner, editor, Superconductivity in Particle Accelerators, pages 191-200. CERN, May 1996. CERN 96-03.

[35] P. Kneisel. Preliminary experience with "in-situ" baking of niobium cavities. In Krawcyk [36], pages $328-335$.

[36] F. Krawcyk, editor. Proceedings of the 9th Workshop on RF Superconductivity, volume I+II, Santa $\mathrm{Fe}$, November 1999. LANL.

[37] V. Palmieri, editor. Proceedings of the 8th Workshop on RF Superconductivity, volume I+II, Abano terme, October 1997. INFN. 